\title{
Stretchable and multifunctional strain sensors based on 3D graphene foams for active and adaptive tactile imaging
}

\author{
Minxuan $\mathrm{Xu}^{1,2}$, Feng $\mathrm{Li}^{1}$, Zhenyun Zhang ${ }^{1}$, Tao Shen ${ }^{1}$, Qian Zhang ${ }^{1}$ and Junjie $\mathrm{Qi}^{{ }^{*}}$
}

\begin{abstract}
The highly developed flexible electronics puts forward higher requirements for the stretchable strain sensors with excellent multiple performances. Herein, a simple and economical fabrication strategy is adopted to obtain a new strain sensor based on Ecoflex rubbers, three-dimensional (3D) graphene foams (GrF) and modified silicone rubber (MSR). The device possesses high stretchability (tolerable strain up to $100 \%$ ) with a variety of capabilities, such as pressure and strain sensing, strain visualization and straincontrolled heating. The GrF with excellent electrical property and MSR with ideal mechanical property endow the sensor with a wide sensing range (up to $100 \%$ strain and $66 \mathrm{kPa}$ stress), high sensitivity (gauge factor of 584.2 within the strain range of $80 \%-100 \%$ and sensitivity of $0.183 \mathrm{kPa}^{-1}$ in $5-10 \mathrm{kPa}$ ) and long cycle life (more than 10,000 cycles) for pressure/ strain sensing. In addition, the temperature of the device can be increased $35^{\circ} \mathrm{C}$ in 5 min under $5 \mathrm{~V}$. Based on this, the deformation is visible to the naked eyes by the color conversion of thermochromic MSR. The soft and reversible strain sensor can be served as the electronic skin (e-skin) for real-time and high accuracy detecting of electrophysiological stimuli, a wearable heater for thermotherapy or body warming and even intelligent visual-touch panel.
\end{abstract}

Keywords: graphene foams, stretchable, strain sensors, multifunctional

\section{INTRODUCTION}

Stretchable strain sensors are receiving growing attention and have great potential for future e-skin [1-3]. Effective transductions such as piezoelectricity [4], capacitance [5], and piezoresistivity [6] are widely used in the stretchable strain sensors, to convert external stimuli into analog electronic signals. The self-powered piezoelectric devices have an absolute advantage in energy consumption, but it is undeniable that their applications are narrow due to failing detection of static strain [7,8]. The major advantage of the capacitive sensors is the characteristic of high strain sensitivity for the detection of a static force with low-power consumption $[9,10]$. Unfortunately, the uneven distribution of the electric field at the edge of the polar plates, which is called edge effect, always results in the nonlinear effect and the decrease of sensitivity [11]. Compared with the above two, the piezoresistive sensors are more suitable for e-skin due to their high performance and stable readout [12].

Great progress has been achieved on piezoresistive stretchable strain sensors, which focuses on the optimization of sensors interfaced with an electronic readout. Such as, resistive devices are designed with unique hierarchical structures [13,14] and coalescent structures [15] to achieve a response time shorter than $100 \mathrm{~ms}$ (blinking speed of human), or more destructive points by making the surface micro-structured to feature an ultrahigh gauge factor (GF) higher than $500[16,17]$, or special ultraelastic composites to broaden the sensing range from $0.01 \%$ [18] to $400 \%[19,20]$. However, these excellent properties are improved individually; many challenges still need to be overcome to realize stretchable strain sensors with full-excellent performance [21]. Most of the recent stretchable strain sensors only feature single function, which makes it poor competitive in advanced eskin [22]. And a few of multifunctional strain sensors are commonly manufactured by complicated production processes $[23,24]$ with high cost.

Herein we fabricate a high-performance and multi-

\footnotetext{
${ }^{1}$ State Key Laboratory for Advanced Metals and Materials, School of Materials Science and Engineering, University of Science and Technology Beijing, Beijing 100083, China

${ }^{2}$ School of Materials and Environmental Engineering, Hangzhou Dianzi University, Hangzhou 310018, China

* Corresponding author (email: junjieqi@ustb.edu.cn)
} 

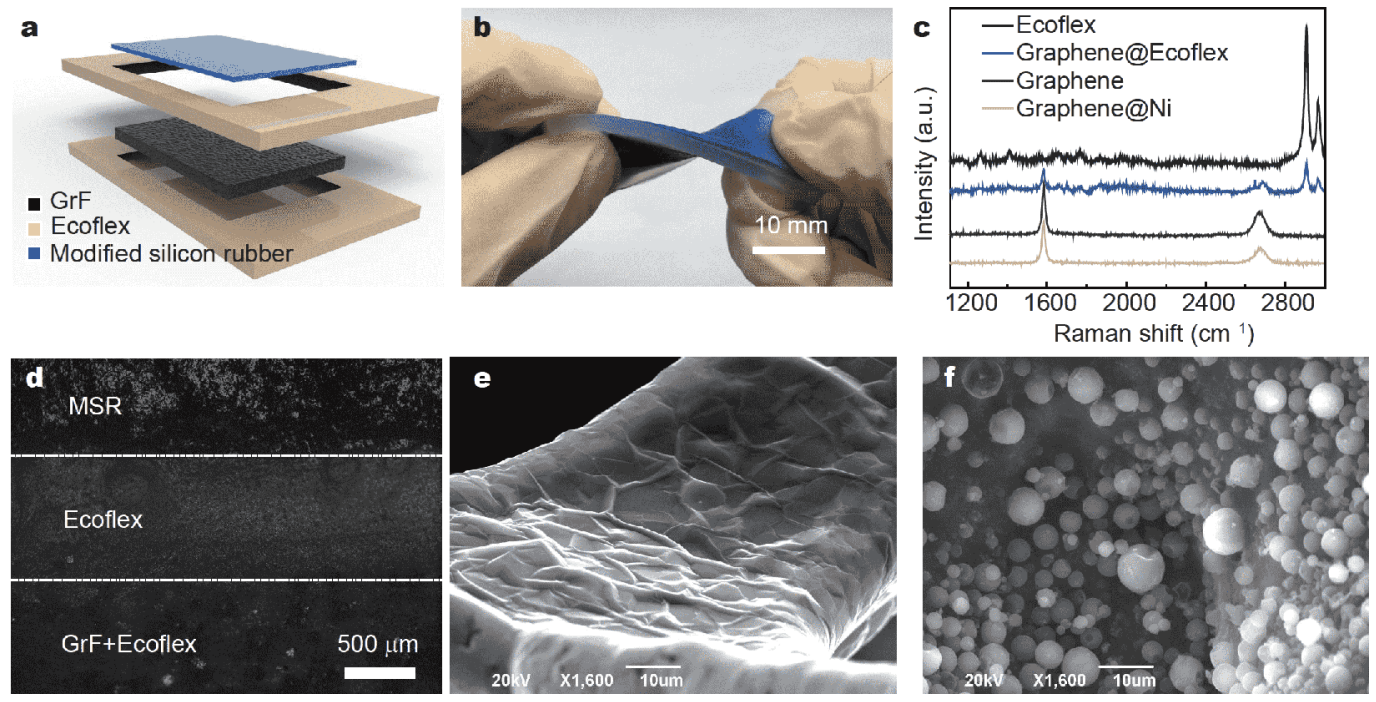

Figure 1 Preparation and characterization of the stretchable strain sensor based on MSR/GrF/Ecoflex composite. (a) Schematic illustration of the strain sensor. (b) Photograph of a torsional strain sensor. (c) Raman shift of pure Ecoflex, pure GrF before and after etching, and the composite of the two. (d) Optical image of the cross-section of the strain sensor. (e) SEM image of the GrF after etching. (f) SEM image of the upper MSR layer.

functional sensor based on Ecoflex rubbers, threedimensional (3D) graphene foams (GrF). The stretchable sensor can be used to detect the pressure up to $66 \mathrm{kPa}$, and exhibits a high sensitivity of $0.183 \mathrm{kPa}^{-1}$ in $5-10 \mathrm{kPa}$. In addition, the sensor possesses a high GF of 22.1 in a wide linear strain region of $0 \%-60 \%$, and the GF increases up to 584.2 in strain range of $80 \%-100 \%$. The ideal sensing properties are attributed to the excellent electric property of GrF and tuned mechanical property by combining with MSR layer. The top silicone rubber modified by the thermochromic materials (TM) makes the strain sensor able to spatially visualize the applied strain, and even serve as a strain-controlled joule heating. The multifunctional sensors are expected as promising components for smart sensing devices, including but not limited to intelligent e-skin, personal healthcare, and visual-touch panel.

\section{RESULTS AND DISCUSSION}

Fig. 1a schematically illustrates the structure of interactive color changeable integrated system with strain detecting, displaying and sealing parts. As shown in Fig. 1b, the obtained strain sensor can be deformed integrally when applied deformation such as torsion. The front and back photographs of the fabricated strain sensor are shown in Fig. S1 (Supplementary information). Raman spectra $(\lambda=633 \mathrm{~nm})$ of 3D GrF, Ecoflex and the assembled GrF/ Ecoflex are shown in Fig. 1c. Two characteristic peaks of GrF at 1,583 and $2,668 \mathrm{~cm}^{-1}$ correspond to $\mathrm{G}$ and $2 \mathrm{D}$ band, respectively. The G-band is assigned to the firstorder scattering of the $\mathrm{E}_{2 \mathrm{~g}}$ mode, and the 2D-band is attributed to the double phonon resonance related to the stacking degree of graphene [25]. The integrated intensity ratio of $2 \mathrm{D}$ and $\mathrm{G}$ bands $\left(I_{2 \mathrm{D}} / I_{\mathrm{G}}=0.63\right)$ also suggests the $3 \mathrm{D} \mathrm{GrF}$ are few-layered [26]. In addition, the characteristic peaks have no significant changes before (canary yellow line) and after (bottle green line) etching. The black line of pure Ecoflex shows two characteristic peaks at 2,908 and 2,968 $\mathrm{cm}^{-1}$. The peaks attributed to the 3D $\mathrm{GrF}$ and Ecoflex also can be found in the blue line that represents the composite structure GrF/Ecoflex (Fig. 1d and Fig. S2). That means the property and structure of $\mathrm{GrF}$ and Ecoflex do not change during the combination process.

The optical image of the cross-section of a fabricated MSR/GrF/Ecoflex device is displayed in Fig. 1d. The MSR layer is on the top of the GrF/Ecoflex layer, and there is a thin layer (100 $\mu \mathrm{m}$ in thickness) of Ecoflex formed by seepage in the middle. Scanning electron microscopy (SEM) analysis was used to characterize the morphology and microstructure of the integrated system. As shown in Fig. 1e and Fig. S3, the highly interconnected 3D graphene network consists of a lot of $200-500 \mu \mathrm{m}$ macropores and the skeletons are wrinkled surface. After assembled with the Ecoflex component, which is shown in Fig. S4b, these holes are filled with liquid epoxy resin precursors to maintain recovery under large deformation. As shown in Fig. 1f, the thermochromic particles in the 
silicone rubber are spherical with regular shape and uniform size (diameters $\leq 10 \mu \mathrm{m}$ ). In addition, the particles uniformly disperse in the silicone rubber. Fig. S4a shows that the interface between MSR layer and $\mathrm{GrF} /$ Ecoflex layer is neat and seamless. The thermochromic particles are organic compounds, and the molecular structure of the organic compound can be changed at a certain temperature due to the electron transfer, so that the color change can be realized. Therefore a visualized color change will appear when the strain causes exceeding a the temperature exceeding a particular value.

Fig. S5 indicates the mechanical properties of the MSR layer with different mass ratio of TM, pure Ecoflex and the composite. The tensile limit of MSR layer is the highest when the mass ratio of TM is $10 \%$. And this MSR shows an ultimate stress of $86.2 \mathrm{kPa}$ at a stretch of $709.8 \%$, whereas the Ecoflex ruptures at a stress of $268.9 \mathrm{kPa}$ and a stretch of $528.2 \%$. As a result, the MSR/ $\mathrm{GrF} /$ Ecoflex composite ruptures at a stress of $186.8 \mathrm{kPa}$ and a stretch of $601.7 \%$. It is reported that the GrF aids in load-bearing, increasing the ultimate tensile strength and ductility while composited with the elastic polymer [27]. Therefore, the stretchability and strength of the assembled device are combination of the two elastomer and closer to Ecoflex.

\section{Compressive pressure sensing}

The electrical properties under pressure is evaluated in an intelligent information acquisition system containing a digital source meter, a semiconductor characterization system and a force gauge installed at the computer-controlled movable stepper motor, which is shown in Fig. S6. A thin glass slide with the size of $150 \mathrm{~mm}^{2}(10 \mathrm{~mm}$ $\times 15 \mathrm{~mm}$ ) is placed over the sensor in order to ensure constant pressure during loading. The pressure from the glass slide $(0.45 \mathrm{~g})$ is defined as "base pressure" for the sensor, and additional pressure is defined as "applied pressure". Fig. 2a demonstrates the current-voltage $(I-V)$ characteristic of the strain sensor with different pressure from 0 to $6.5 \mathrm{kPa}$. In the process of increasing pressure, the current significantly decreases according to the reduction of network in the GrF, which gives rise to a corresponding conductivity decrease. The results are consistent with the reported work on GrF based strain sensor [28].

To demonstrate the excellent pressure-responsive properties of MSR/GrF/Ecoflex strain sensor, the electronic resistance variation ratios $\left(\Delta R / R_{0}=\left(R_{\mathrm{P}}-R_{0}\right) / R_{0} ; R_{0}\right.$ and $R_{\mathrm{P}}$ corresponds to the resistance without and with pressure, respectively) with respect to the compression are investigated. As presented in Fig. $2 \mathrm{~b}$, the pressure sensitivity $(S)$ of the strain sensor is calculated with the measured values [20], which can be defined as $S=\delta(\Delta R /$ $\left.R_{0}\right) / \delta_{\mathrm{p}}$. The relative resistance change curve indicates that the pressure sensitivity decreases gradually due to the increase of pressure. The pressure applied by the force gauge installed at the computer-controlled movable stepper motor is range from $5.26 \mathrm{kPa}(78.9 \mathrm{~g})$ to $40.2 \mathrm{kPa}$ $(603.4 \mathrm{~g})$. The pressure sensitivity is calculated to be $0.183 \mathrm{kPa}^{-1}$ in low-pressure region $(5-10 \mathrm{kPa})$ and $0.031 \mathrm{kPa}^{-1}$ in the high-pressure region $(30-40 \mathrm{kPa})$. To obtain the pressure detection limit of the stretchable strain sensor, the weight has been used to provide pressure less than $5 \mathrm{kPa}$ or more than $40 \mathrm{kPa}$. As a result, the device detects the lowest level of $4 \mathrm{kPa}$ and highest level of $66 \mathrm{kPa}$, as shown in Fig. S7.

The change of sensitivity is due to the nonlinear relationship between stress and stress-caused deformation, which has been reported previously in the similar composites of Ag NW/Pyy [29], rGO/PI [30], and SWNT/ PDMS [31]. These compressive stress-strain curves imply that when the pressure increases, the effective deformation caused by stress will increase linearly and slowly. As long as the strain exceeds a certain limit, the further increase of the deformation requires much greater stress. In other words, the same stress can only produce smaller deformation than before, which results in the decreasing resistance response at higher applied pressure. Among the resistance-type strain sensors, our pressure sensitivity $\left(0.183 \mathrm{kPa}^{-1}\right)$ is favorably comparable with recent values of other nanocomposite sensors $\left(0.09-0.33 \mathrm{kPa}^{-1}\right)[20,29]$, and higher than that of the previously reported one $\left(0.001-0.088 \mathrm{kPa}^{-1}\right)[24,32]$.

Fig. 2c shows the electrical cycling responses of MSR/ $\mathrm{GrF} / \mathrm{Ecoflex}$ strain sensor under various dynamic pressure with frequency of $0.1 \mathrm{~Hz}$. The stable and continuous responses could be observed at various pressure. In addition, the strain sensor is subjected to different degrees of pressure and held at each pressure for $5 \mathrm{~s}$, exhibiting small drop at large strain as the pressure holds on. It may be attributed to the acceleration and viscoelastic nature of the elastic polymer [33]. On the other hand, as presented in the recent literature, the deformation mechanism in compression stress is GrF branch bending and branch wall elastic depression, which is not on account of the exceptionally high in-plane mechanical properties of $\mathrm{GrF}$ [34]. And the damage localization is avoided in GrF by dispersing the force of impact over a wider area, making them impact tolerable [35]. In other words, the special graphene frame will weaken the compressive deformation 

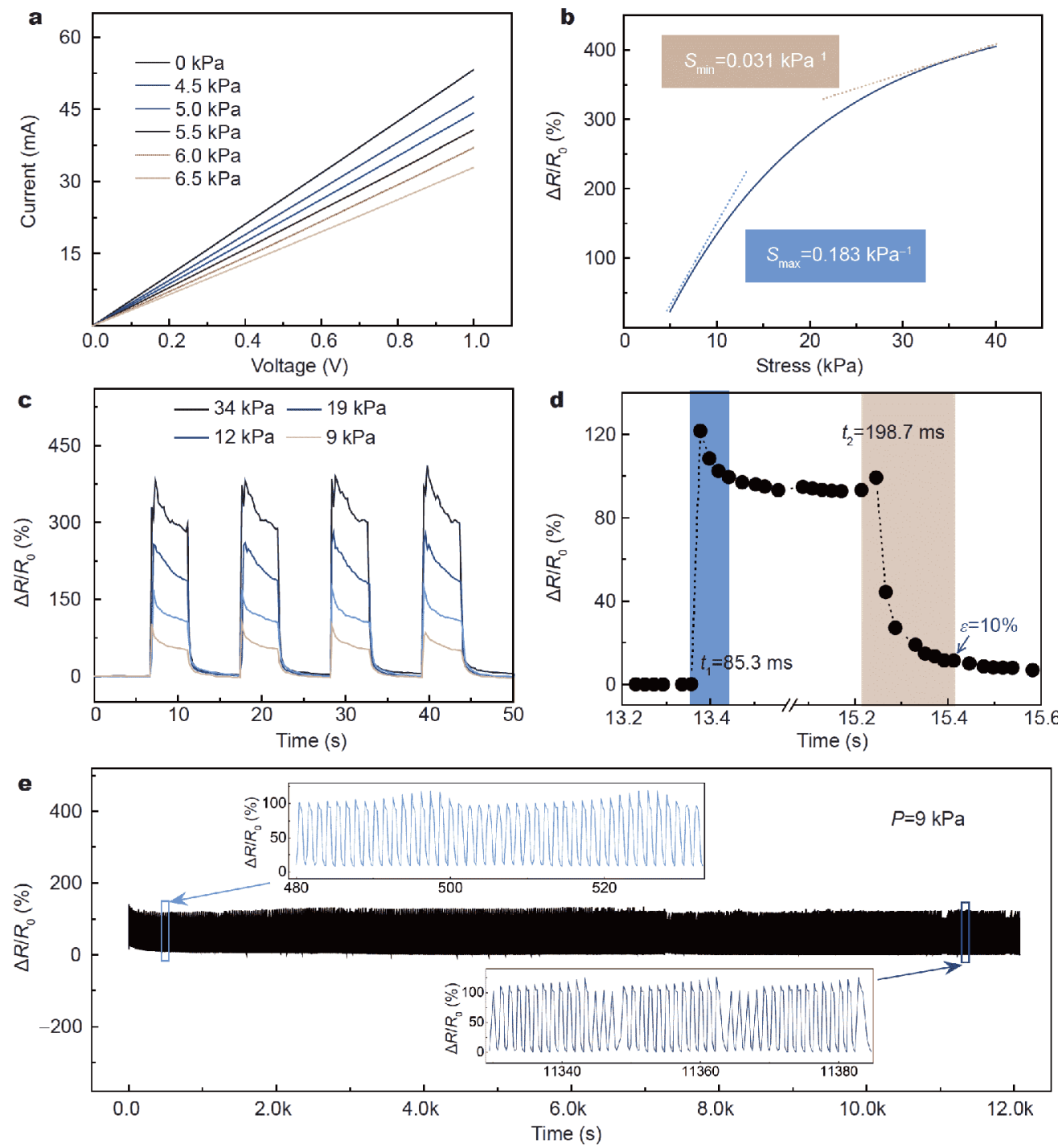

Figure 2 Application of the multifunctional device as compressive stress detection. (a) Current-voltage of the strain sensor under different applied pressure. (b) Sensitivities of strain sensor during the pressure from 0 to $40 \mathrm{kPa}$. (c) Multiple-cycle tests of change in resistance with different applied pressure. (d) Real-time fast response and release of the strain sensor upon $9 \mathrm{kPa}$ pressure. (e) Cycling stability test of strain sensor under repeated applied pressure of $9 \mathrm{kPa}$ for 10,000 cycles (1.2 s for each cycle).

because of dispersion, resulting in a slight decline in the electrical signal of the device. When pressure is up to $9 \mathrm{kPa}$, the variation ratio of resistance is $102.5 \%$. This relationship is confirmed to be linear. With the increase of applied pressure from 12 to $34 \mathrm{kPa}$, the change in resistance variation ratios for strain sensor is about $164.5 \%$, $254 \%$ and $382.8 \%$, respectively. Fig. $2 \mathrm{~d}$ illustrates the single response curve of the strain sensor at a pressure of $9 \mathrm{kPa}$. The instant stimuli on the sensor exhibits a fast response time of $85.3 \mathrm{~ms}$ and a little slower recovery time of $198.7 \mathrm{~ms}$ (the relative resistance change reduced from
$100 \%$ to $10 \%)$. Then it may take seconds for the response curve to fall from $10 \%$ to $0 \%$. To further investigate the stability of the strain sensor, the relative resistance changes of the device are measured when repeatedly loading/unloading an applied pressure of $9 \mathrm{kPa}$ for more than 10,000 cycles $(1.2 \mathrm{~s}$ for each cycle). The cycling stability test is carried out by the force gauge installed at the computer controlled movable stepper motor, in which the tip of force gauge does not touch the entire device, but loads on the thin glass slide over the device. The result (Fig. 2e) reveals that the pressure sensor exhibits high 

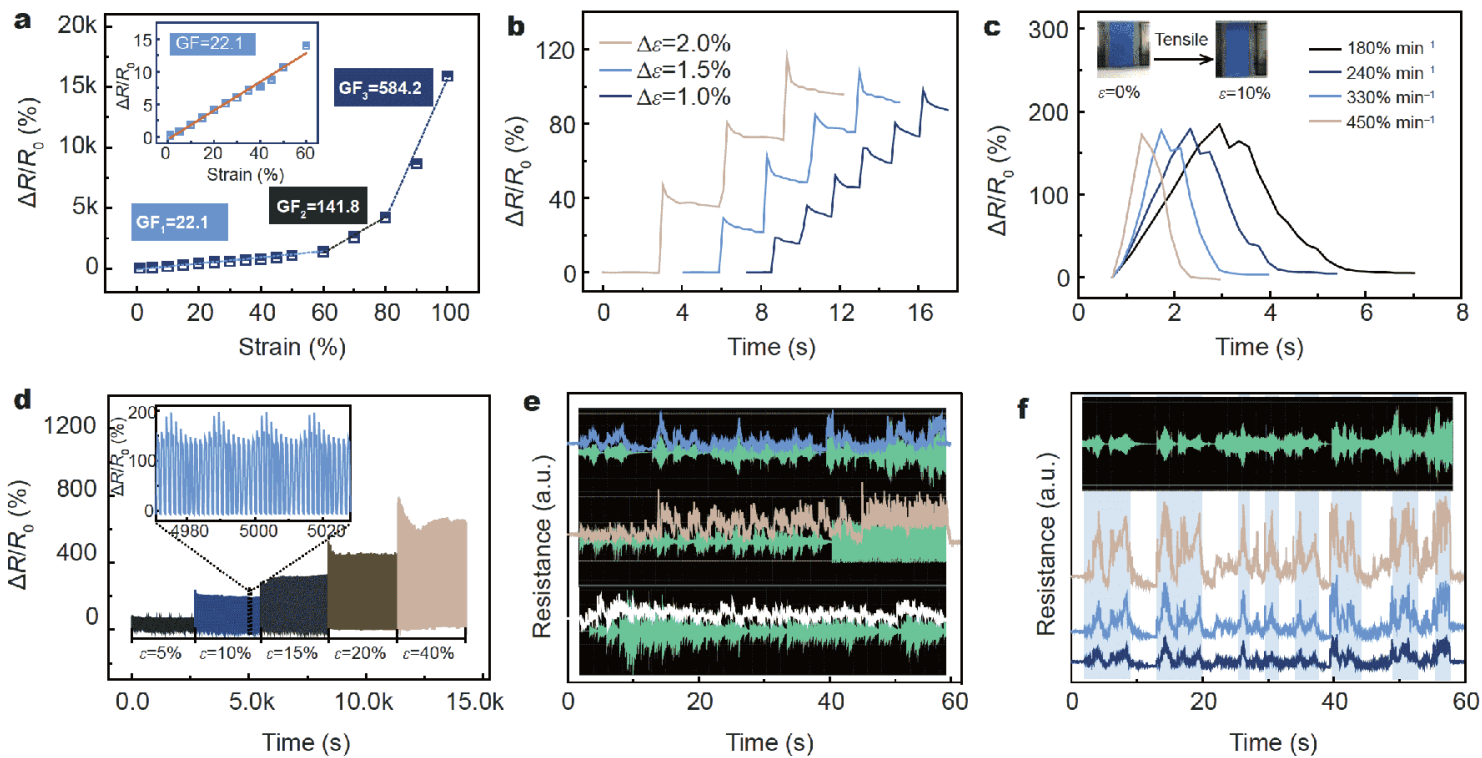

Figure 3 Application of the multifunctional device as tensile strain detection. (a) Typical relative resistance-strain curve of a strain sensor within $100 \%$ strain; the inset shows the curve within $60 \%$ strain. (b) The time-resistance curves for repeated step-by-step stretching at different rates. (c) Variation of the resistance of the MSR/GrF/Ecoflex composite with the strain of $10 \%$ under different strain rates from $180 \% \mathrm{~min}^{-1}$ to $450 \% \mathrm{~min}^{-1}$. (d) Reliability test of the sensor under repeated cycles of stretching and releasing at different values with partial enlarged details in insets. (e) Recognition of three songs $(60 \mathrm{~dB})$ played by mobile phone for the strain sensor (the green curves in the background panels are the sound wave profiles). (f) Zoomed signals of the sensor corresponding to the same vibration audio at different decibels $(60,80$, and $100 \mathrm{~dB})$.

stability and durability.

\section{Tensile strain sensing}

The MSR/GrF/Ecoflex strain sensor is also able to detect the tensile deformation. In situ SEM observation reveals that the high strength in tension is due to GrF branch alignment that enables branches to utilize the high inplane mechanical properties of GrF [34]. After composited with the elastic polymer, the characteristic singly deflected cracks are distinguished by its deflection through one side of the interface of GrF when tension applied. Such behavior serves as evidence of the highenergy dissipation and absorption capability provided by the reinforcement in the matrix [36].

To test the performance of the strain sensor, the resistance variation ratios $\left(\Delta R / R_{0}=\left(R_{\varepsilon}-R_{0}\right) / R_{0} ; R_{0}\right.$ and $R_{\varepsilon}$ corresponds to the resistance without and with tensile, respectively) monitored by stretching strains are investigated. Here, the sensitivity is indicated by the GF [16], which is defined as GF $=\delta\left(\Delta R / R_{0}\right) / \delta \varepsilon$. As shown in Fig. $S 8, \varepsilon$ is the stretch strain of the device $\left(\varepsilon=\left(L-L_{0}\right) / L_{0}\right.$, where $L_{0}$ and $L$ are the lengths of the sensing GrF before and after stretching). The relative resistance changes of the strain sensors upon stretching to different strains are shown in Fig. 3a. As the applied strain $\varepsilon$ increases from $0 \%$ to $60 \%$, the relative resistance changes $\left(\Delta R / R_{0}\right)$ slowly and linearly with the GF calculated to be 22.1. The relative resistance-strain curve, as shown in the inset of Fig. 3a, demonstrates that the strain sensor has a broad linear sensing range (up to $60 \%$ ), which is high enough for the film to be used as a wearable physical sensor in personal healthcare (primary sensing target: $\varepsilon<55 \%$ ). When the tensile strain exceeds $60 \%$, the resistance variation ratios increase exponentially with strain. Fig. S9 shows the GF of the strain sensor at strains $>60 \%$, which is calculated to be 141.8 ( $\varepsilon: 60 \% \sim 80 \%$ ), and 584.2 ( $\varepsilon: 80 \%$ $\sim 100 \%)$. The results indicate the as-prepared stain sensors possess higher piezoresistive responses than previously reported $\mathrm{GrF}$ based strain sensors, such as GrF/PDMS (GF 29) [28], GrF/PI (GF 5) [30], GrF/PU (GF 28) [37], and $\mathrm{GrF} /$ rubber (GF 35) [38].

As shown in Fig. 3b, we also examined the resistance variation of the MSR/GrF/Ecoflex strain sensor by stretching the sensor to $6 \%$ step by step (the step strain $\Delta \varepsilon$ was increased from $1 \%$ to $2 \%$ ). It indicates the excellent elasticity and stable stretch response of the strain sensor based on 3D GrF. Considering the acceleration-dependence of the strain sensor is one of the major character- 
istics, herein, we investigate the responses in resistance of the strain sensor at different loading frequency ranging from $180 \% \mathrm{~min}^{-1}\left(\Delta R / R_{0}=184.4 \%\right)$ to $450 \mathrm{~min}^{-1}(\Delta R /$ $R_{0}=171.7 \%$ ), as shown in Fig. $3 \mathrm{c}$. Only $12.7 \%$ drop is observed at the applied strain levels, which further reveals the reliable and stable response of the MSR/GrF/Ecoflex strain sensor.

As shown in Fig. 3d, the relative resistance changes of the device fluctuate slightly within a certain range and the cycling performance shows periodic fluctuation during the repeated stretching to $5 \%, 10 \%, 15 \%, 20 \%$ and $40 \%$ more than 2,000 cycles, respectively ( $1.4 \mathrm{~s}$ for each cycle). Fig. S10 characterizes the device before or after the stretch cycling test. As shown in Fig. S10a and b, there is no obvious change in the appearance of the device after stretching for 10,000 cycles. The high magnification optical image (Fig. S10d) shows the Ecoflex interlayer is slightly thinner than the pristine one (Fig. S10c), which corresponds to the small amplitude of the signals. However the interfaces between the multi-components display a stable combination, which indicates the strain sensor can reversibly restore its original state. As shown in Fig. S5b, combining Ecoflex with MSR leads to the decrease of mechanical stiffness, which results in the reduction of the Young's modulus and increase of the recovery time. However, when the recovery time (about a few seconds) of the device is longer than the interval time (1.2 or $1.4 \mathrm{~s}$ ) of the pressure/strain applied, the device will take several cycles to return to the initial state $(\varepsilon=0 \%)$, which is shown as a periodic fluctuation in the relative-resistance curve.

The unique multi-layered strain sensor can also be adopted to detect acoustic vibration with ultrahigh frequency $(5-10 \mathrm{kHz})$, which requires the sensors with ultrahigh sensitivity. The sensor is attached to the receiver of the phone to record the vibration produced by the mobile phone music ("half-moon serenade", "seen you again" and "the Moon over a fountain"). As shown in Fig. $3 \mathrm{e}$, the tiny vibrational forces from music can be accurately detected and the three songs can be distinguished by the distinct electrical resistance curves obtained under the same decibel $(60 \mathrm{~dB})$. Note that the background panels show the sound wave profiles of the ringtones are highly consistent with the test curves of each song. Fig. $3 \mathrm{f}$ further shows the signals of the sensor response corresponding to the same song ("half-moon serenade") under different decibels $(60,80$ and $100 \mathrm{~dB})$. The obtained three curves have the same characteristic peaks and valleys, which are close to the sound wave profiles shown in the top panel. Therefore, it can be concluded that the successful detection of various music songs is beneficial for the application of voice recognition with the strain sensors.

The resistance signal of the stretchable MSR/GrF/Ecoflex strain sensor is sensitive to the tiny vibration caused by blood pressure and other human motions. Fig. 4 presents the real-time change in resistance for the wearable strain sensor attached on the wrist and neck of a human being. The human pulse waveforms (AWP) in Fig. 4a clearly displayes the characteristic peaks including percussion (P), tidal (T), and diastolic (D) peaks. Fig. 4b reveals the obtained biphasic waveform of the jugular venous pulse (JVP), where we can easily resolve three upward deflections named atrial contraction (A), ventricular contraction (C), and atrial venous filling (V) [39]. The fast response demonstrates that the MSR/GrF/Ecoflex strain sensor can identify the subtle differences in blood pulses, indicating its potential to serve as a wearable diagnostic device to provide detailed clinical information. As shown in Fig. 4c, since the response is almost distinct and stable with high signal-to-noise ratio, the strain sensor can record the subtle characteristic signals generated from JVPs even when they are complicated by trachea (breathing). As shown in Fig. 4d, other motion such as saliva swallowing showing unique vibrational waveforms also can be identified using the strain sensor. Therefore, it can be concluded that the successful detection of various tiny forces is beneficial for the application of human-motion monitoring with the MSR/GrF/Ecoflex strain sensors.

\section{Strain-controlled heating}

Measurement setup is schematically shown of in Fig. 5a. A direct current power for voltage applying and a digital source meter for resistance test are connected to the electrodes of the device. While the real-time temperature is measured by a temperature tester, which touches the device surface with a test probe. The computer controls the stepper motor moving to apply a tensile deformation on the device. The relative resistance changes $\Delta R / R_{0}$ of the strain sensor at different temperatures are carefully examined. As shown in Fig. $5 \mathrm{~b}$, the $\Delta R / R_{0}$ of the strain sensor (canary yellow line) decreases from $0 \%$ to $-24 \%$ with the decrease of temperature from 50 to $14^{\circ} \mathrm{C}$. The resistivity decrease indicates the opposite trend to the reported work on pure GrF [40]. That is probably caused by the structural damage of GrF (wrapped inside the hot swelling Ecoflex rubber) at high temperature and the damaged structure becomes restored during the cooling process.

Furthermore, the electric heating behavior of MSR/ 

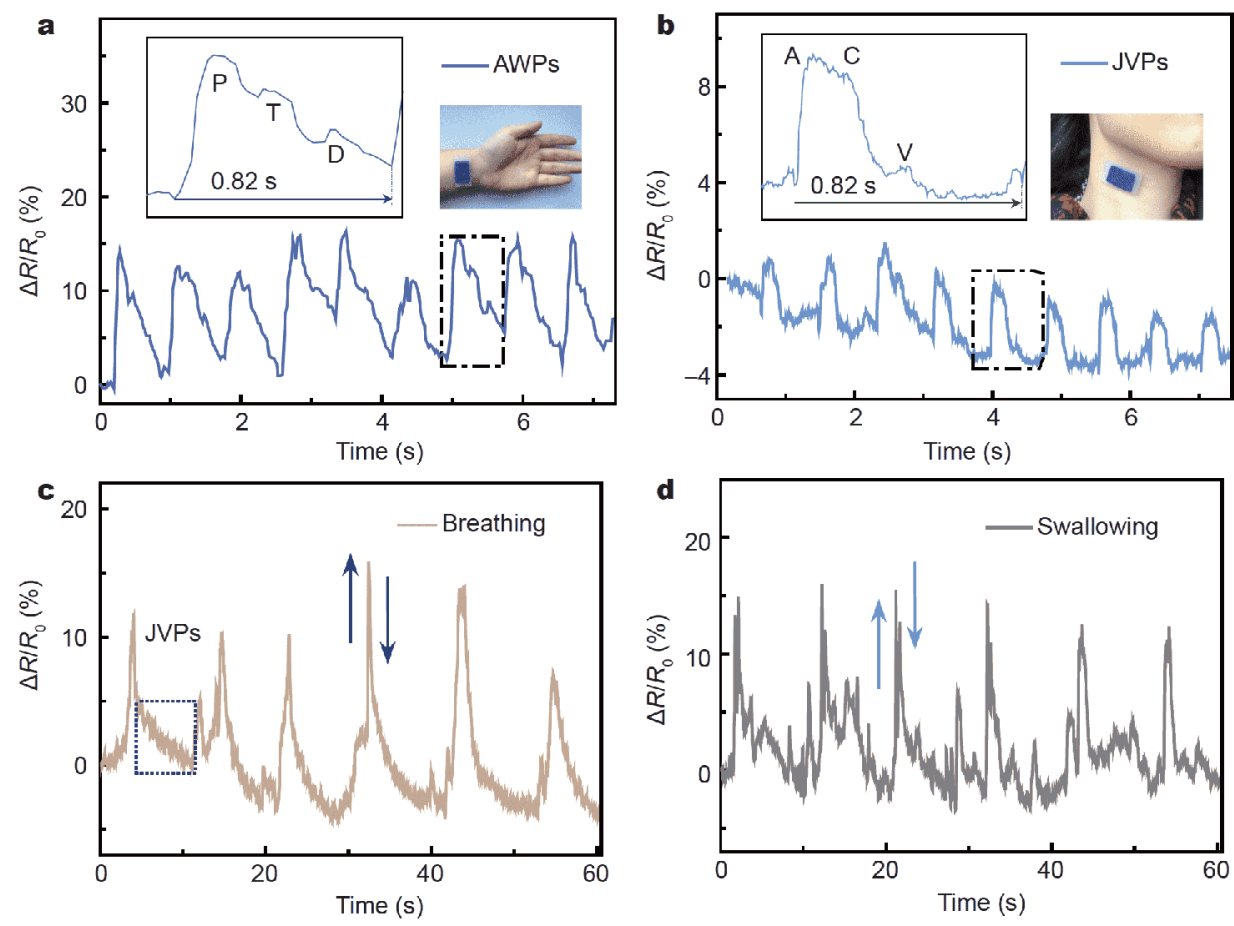

Figure 4 Application of the multifunctional device in human motion detection. (a) Real-time and in situ AWPs measurement with the skinattachable strain sensor attached on the wrist. The inset shows the zoomed waveform. (b) Measurement of JVPs with strain sensors attached on the neck. The inset is the zoomed waveform. (c) Relative resistance changes versus time for the strain sensor during breathing. (d) Relative resistance changes versus time for the strain sensor during saliva swallowing.

GrF/Ecoflex composite was also investigated by applying various voltages. As shown in Fig. $5 c$, the final temperature of the strain sensor under $1,2,3,4$ and $5 \mathrm{~V}$ for $5 \mathrm{~min}$ was around $14.7,19.5,25.8,36.0$ and $49.9^{\circ} \mathrm{C}$, respectively. Under applied voltage, the surface temperature of the strain sensor increases quickly within the first two minutes and then reaches a relatively slow heating stage. At the initial stage, most of the joule heat converted from the current is used to heat up the strain sensor, while only a small portion is dissipated into the surroundings. While the temperature rises, the heat dissipation into the ambient increases, and the resistance of the sensor also increases according to previous test results (Fig. 5b), both of which result in the slowing down of the heating rate. In principle, the thinner the sandwiched Ecoflex between the MSR and the GrF is, or the more seamless the interface between the MSR layer and GrF/Ecoflex layer is, the less the heat losses, which reduces heating time and increases heating temperature at the initial stage. Because the power of a device is related to the working voltage and current, the heating temperature of the strain sensor can be controlled by adjusting the resistance while the voltage is constant, which means the heating temperature of our device can be regulated not only by voltage [41] but also by strain.

As indicated by Fig. 5d, under working voltage of $5 \mathrm{~V}$ for $5 \mathrm{~min}$, the final temperature of the strain sensor under $0 \%, 20 \%, 40 \%$, and $60 \%$ is around $49.9,38.9,30.8$ and $21.8^{\circ} \mathrm{C}$, respectively. So the strain-control can still work normally when the voltage regulator fails. This is a double guarantee of the accurate control of the heating temperature. In addition, as the voltage does not need to change during changing the heating temperature, the $1.5 \mathrm{~V}$ battery can be used as the power supply, which will make the heater more portable. As shown in Table S1, the GrF-based strain sensors possess a broadened applications compared with the previously reported strain sensors based on nano- and micro- materials, which fail to possess multiple functions including pressure/strain sensing and joule heating at the same time.

\section{Pressure and strain visualization}

Due to the relationship between colour and temperature, temperature and resistance, resistance and strain, the strain sensor displays the strain in different colour, namely strain visualization. Fig. 6a shows the changes in 

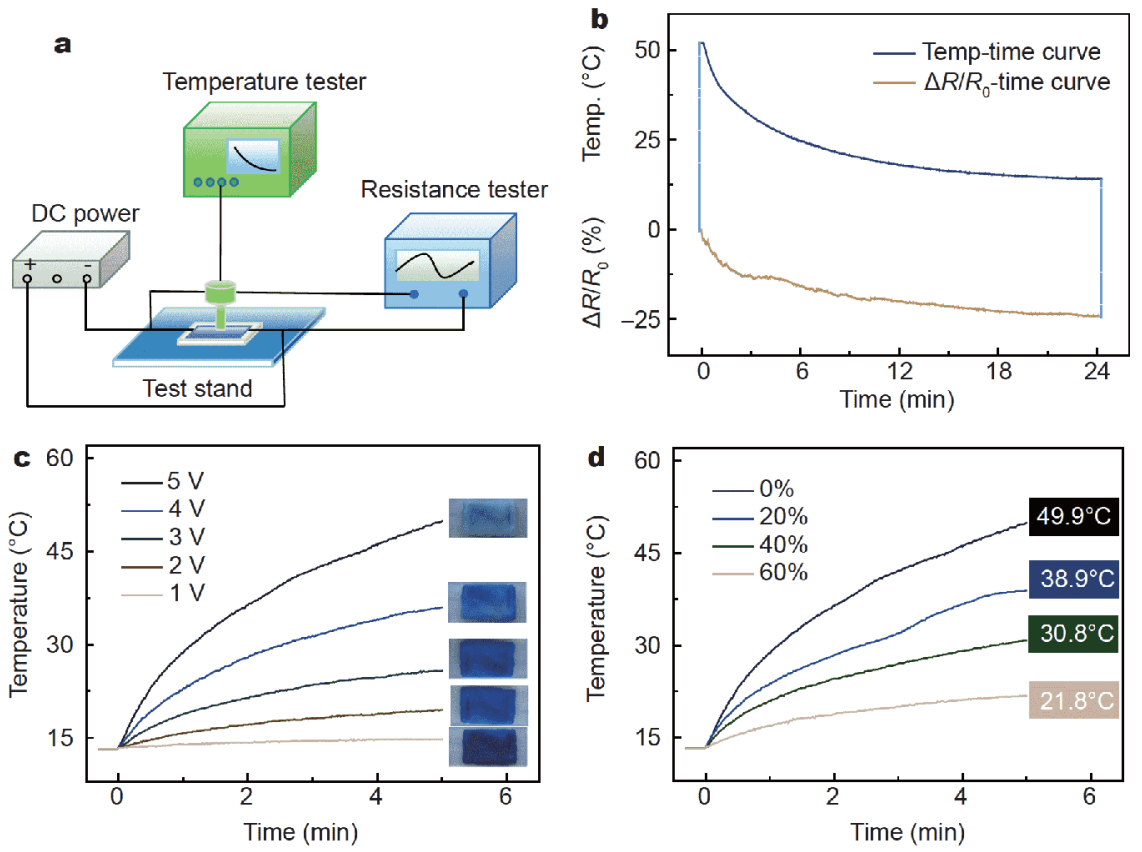

Figure 5 Application of the multifunctional device in strain-controlled heater. (a) Schematic of measurement setup. (b) Relative electrical resistance of the strain sensor and temperature curve with time. (c) Heating behavior of the MSR/GrF/Ecoflex composite under various working voltages (the inset is the photographs). (d) Temperature-time curves of the MSR/GrF/Ecoflex composite under various strain states.

$\Delta R / R_{0}$ (canary yellow line) and temperature (dark purple line) in accordance with the strain from $0 \%$ to $60 \%$. With the increase in strain, the temperature of the sensor decreases, which corresponds to the colour changing from light to dark blue. The colour of the strain sensor distinguishes the magnitude of the applied strain, and indicates the visual display of the applied strain in a quantitative manner. Fig. $6 \mathrm{~b}$ shows the ability to detect and visualize the distribution of panel strain signals, and the strip-like devices is magnified into a larger square one with the effective MSR of $50 \mathrm{~mm} \times 50 \mathrm{~mm}$. The MSR layer shows light blue as the working voltage is maintained at $5 \mathrm{~V}$. The three balls (each weight $3.8 \mathrm{~g}, 10^{\circ} \mathrm{C}$ ) are placed on the MSR in turn and then taken away. The real-time response curve increases during loading balls and decreases while removing them. Notably, the strain sensor responds rapidly at each loading/unloading and the resistance remains identical under the same pressure state. Interestingly, when the ball is removed, the dark blue mark will be left on the MSR layer due to the colour change generated by the temperature difference. As shown in the optical images (Fig. 6b), this color contrast can last for a few seconds and shows the pressure distribution on the panel.

Application of the MSR/GrF/Ecoflex-based strain sensor for active and adaptive tactile imaging is explored.
The as-formed device is fixed on the computer-controlled movable stepper motor and kept horizontal. Fig. $6 \mathrm{c}$ and $\mathrm{d}$ demonstrate the electrical response behaviors of the strain sensor in the process of words writing. The resistance curve indicates the strain sensor exhibits high sensitivity and distinct patterns while writing different words such as "USTB" (purple line) and "NANO" (black line). To further investigate its repeatability under the tensile states, the word "USTB" and "NANO" are recorded at $25 \%$ stretch strain (yellow and blue line). Compared to the initial state $(0 \%$ stretch strain), it is clear that the obtained $R$ - $t$ curves have similar characteristic peaks and valleys when writing the same words. Fig. $6 \mathrm{c}$ and d show a series of photographs of writing words under $0 \%$ stretch strain. The MSR, which served as display layer, exhibits a light blue color at the working voltage $5 \mathrm{~V}$. After writing the words ("USTB" and "NANO") with a $10^{\circ} \mathrm{C}$ metal tip, the words will show a dark blue color. The temperature difference between the metal tip and the MSR layer will cause the discoloration of the internal thermochromic powders. The MSR-based strain sensor provides an interesting and effective method for handwriting recognition through sensitive and fast pressure imaging, which is mainly caused by the combination of 3D graphene networks and thermochromic material. It may be useful for stretchable handwriting panel, which will bring promise 

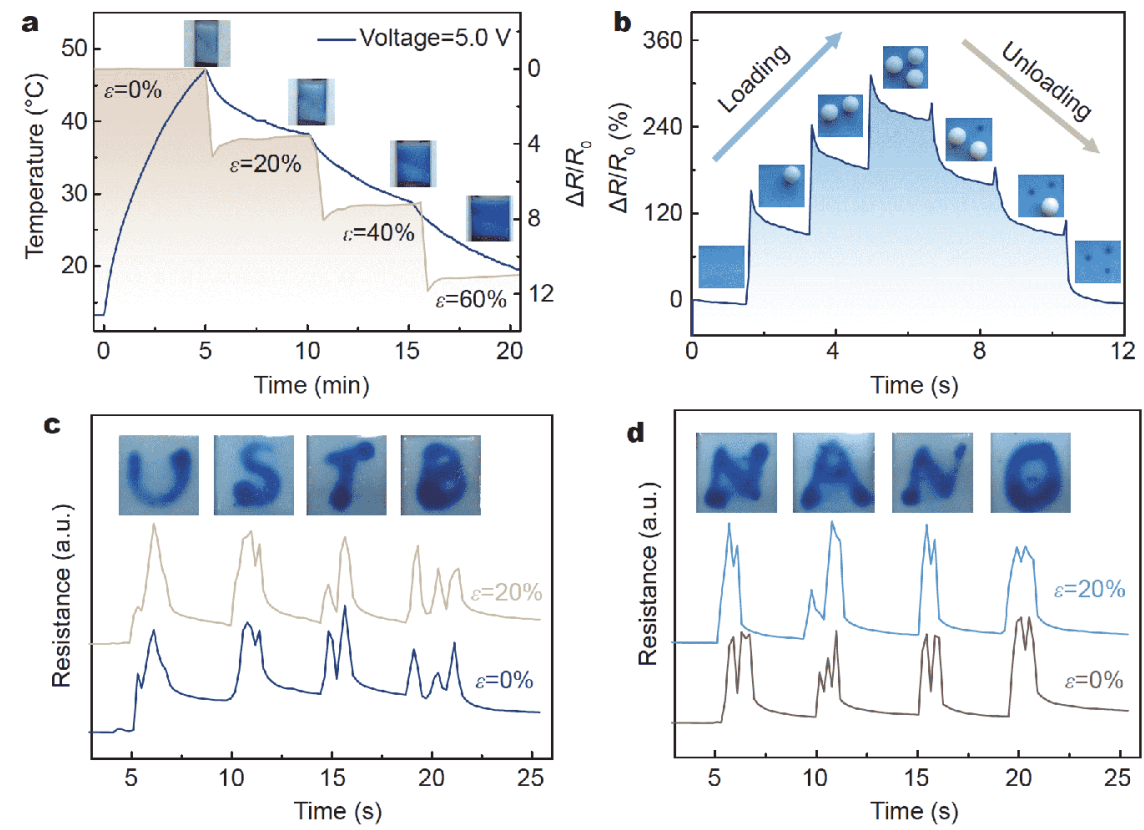

Figure 6 Application of the multifunctional device in strain visualization. (a) Real-time temperature changes corresponding to various applied strains, each maintained for $5 \mathrm{~min}$. (b) The strain distribution for "Loading" and "Unloading" compress applied of balls with different position. (c) Relative resistance changes for the stretchable panel in writing process of "USTB". (d) Relative resistance changes for the stretchable panel in writing process of "NANO". The insets are the photographs, respectively.

for remote control of human-machine interfaces.

\section{CONCLUSION}

In summary, a stretchable strain sensor is assembled with elastic Ecoflex rubber, 3D graphene interconnection conductive network and modified silicone rubber. The graphene network is responsible for detecting the compressive stress and tensile strain. The upper silicone rubber modified by thermochromic material shows the strain distribution according to the temperature difference. The elastic Ecoflex rubber is used to package the device, serving as a supporting substrate. As a result, the developed strain sensor possesses a desirable electrical conductivity with high sensitivity, wide strain range, fast response, and excellent durable stability. The ultrahigh sensitivity and signal-to-noise ratio make the sensor promising in detecting of electrophysiological stimuli and even acoustic vibration recognition. Due to its excellent flexibility and moderate electrical conductivity, the strain sensor is suitable as a wearable heater for thermotherapy or body warming. Remarkably, through the demonstrations of some potential applications, the strain sensor can be used as promising components for smart sensors, including monitoring the various handwriting words and imaging. We believe that the methodologies put forward a novel design and a scalable approach to explore wide- spread applications in multifunctional strain sensors, and may further advance the wearable panel of human-computer interactions.

Received 23 June 2018; accepted 1 September 2018;

published online 25 September 2018

1 Jian M, Wang C, Wang Q, et al. Advanced carbon materials for flexible and wearable sensors. Sci China Mater, 2017, 60: 10261062

2 Liu Y, He K, Chen G, et al. Nature-inspired structural materials for flexible electronic devices. Chem Rev, 2017, 117: 12893-12941

3 Luo CS, Wan P, Yang H, et al. Healable transparent electronic devices. Adv Funct Mater, 2017, 27: 1606339

4 Qi J, Lan YW, Stieg AZ, et al. Piezoelectric effect in chemical vapour deposition-grown atomic-monolayer triangular molybdenum disulfide piezotronics. Nat Commun, 2015, 6: 7430

5 Lipomi DJ, Vosgueritchian M, Tee BCK, et al. Skin-like pressure and strain sensors based on transparent elastic films of carbon nanotubes. Nat Nanotechnol, 2011, 6: 788-792

6 Pan L, Chortos A, Yu G, et al. An ultra-sensitive resistive pressure sensor based on hollow-sphere microstructure induced elasticity in conducting polymer film. Nat Commun, 2014, 5: 3002

7 Huang YA, Ding Y, Bian J, et al. Hyper-stretchable self-powered sensors based on electrohydrodynamically printed, self-similar piezoelectric nano/microfibers. Nano Energy, 2017, 40: 432-439

8 Wang T, Yang H, Qi D, et al. Mechano-based transductive sensing for wearable healthcare. Small, 2018, 14: 1702933

9 Yao S, Zhu Y. Wearable multifunctional sensors using printed stretchable conductors made of silver nanowires. Nanoscale, 2014, 6: $2345-2352$ 
10 Lee J, Kwon $\mathrm{H}$, Seo J, et al. Conductive fiber-based ultrasensitive textile pressure sensor for wearable electronics. Adv Mater, 2015, 27: 2433-2439

11 Wu JJ, Chen CX, Ma TH, et al. Edge effect analysis of capacitive grid sensor based on ansoft. Instrument Technique and Sensor, 2016, 2: 1-4

12 Wang X, Dong L, Zhang $\mathrm{H}$, et al. Recent progress in electronic skin. Adv Sci, 2015, 2: 1500169

13 Jian M, Xia K, Wang Q, et al. Flexible and highly sensitive pressure sensors based on bionic hierarchical structures. Adv Funct Mater, 2017, 27: 1606066

14 Wang Q, Jian M, Wang C, et al. Carbonized silk nanofiber membrane for transparent and sensitive electronic skin. Adv Funct Mater, 2017, 27: 1605657

15 Xia K, Wang C, Jian M, et al. CVD growth of fingerprint-like patterned 3D graphene film for an ultrasensitive pressure sensor. Nano Res, 2018, 11: 1124-1134

16 Liao X, Zhang Z, Kang Z, et al. Ultrasensitive and stretchable resistive strain sensors designed for wearable electronics. Mater Horiz, 2017, 4: 502-510

17 Wang C, Xia K, Zhang M, et al. An all-silk-derived dual-mode eskin for simultaneous temperature-pressure detection. ACS Appl Mater Interfaces, 2017, 9: 39484-39492

18 Wang C, Xia K, Jian M, et al. Carbonized silk georgette as an ultrasensitive wearable strain sensor for full-range human activity monitoring. J Mater Chem C, 2017, 5: 7604-7611

19 Wang C, Li X, Gao E, et al. Carbonized silk fabric for ultrastretchable, highly sensitive, and wearable strain sensors. Adv Mater, 2016, 28: 6640-6648

20 Xu M, Qi J, Li F, et al. Highly stretchable strain sensors with reduced graphene oxide sensing liquids for wearable electronics. Nanoscale, 2018, 10: 5264-5271

21 Trung TQ, Lee NE. Flexible and stretchable physical sensor integrated platforms for wearable human-activity monitoringand personal healthcare. Adv Mater, 2016, 28: 4338-4372

22 Liao X, Liao Q, Zhang Z, et al. A highly stretchable ZnO@fiberbased multifunctional nanosensor for strain/temperature/UV detection. Adv Funct Mater, 2016, 26: 3074-3081

$23 \mathrm{Xu} \mathrm{H}$, Xiang JX, Lu YF, et al. Multifunctional wearable sensing devices based on functionalized graphene films for simultaneous monitoring of physiological signals and volatile organic compound biomarkers. ACS Appl Mater Interfaces, 2018, 10: 11785-11793

24 Hua Q, Sun J, Liu H, et al. Skin-inspired highly stretchable and conformable matrix networks for multifunctional sensing. Nat Commun, 2018, 9: 244

25 Kudin KN, Ozbas B, Schniepp HC, et al. Raman spectra of graphite oxide and functionalized graphene sheets. Nano Lett, 2008, 8: 3641

26 Çelik Y, Flahaut E, Suvacı E. A comparative study on few-layer graphene production by exfoliation of different starting materials in a low boiling point solvent. FlatChem, 2017, 1: 74-88

27 Embrey L, Nautiyal P, Loganathan A, et al. Three-dimensional graphene foam induces multifunctionality in epoxy nanocomposites by simultaneous improvement in mechanical, thermal, and electrical properties. ACS Appl Mater Interfaces, 2017, 9: 3971739727
28 Jeong YR, Park H, Jin SW, et al. Highly stretchable and sensitive strain sensors using fragmentized graphene foam. Adv Funct Mater, 2015, 25: 4228-4236

29 He W, Li G, Zhang S, et al. Polypyrrole/silver coaxial nanowire aero-sponges for temperature-independent stress sensing and stress-triggered Joule heating. ACS Nano, 2015, 9: 4244-4251

30 Qin Y, Peng Q, Ding Y, et al. Lightweight, superelastic, and mechanically flexible graphene/polyimide nanocomposite foam for strain sensor application. ACS Nano, 2015, 9: 8933-8941

31 Wang X, Gu Y, Xiong Z, et al. Silk-molded flexible, ultrasensitive, and highly stable electronic skin for monitoring human physiological signals. Adv Mater, 2014, 26: 1336-1342

32 Tewari A, Gandla S, Bohm S, et al. Highly exfoliated MWNT-rGO ink-wrapped polyurethane foam for piezoresistive pressure sensor applications. ACS Appl Mater Interfaces, 2018, 10: 5185-5195

33 Yamada T, Hayamizu Y, Yamamoto Y, et al. A stretchable carbon nanotube strain sensor for human-motion detection. Nat Nanotechnol, 2011, 6: 296-301

34 Nieto A, Boesl B, Agarwal A. Multi-scale intrinsic deformation mechanisms of 3D graphene foam. Carbon, 2015, 85: 299-308

35 Nautiyal P, Boesl B, Agarwal A. The mechanics of energy dissipation in a three-dimensional graphene foam with macroporous architecture. Carbon, 2018, 132: 59-64

36 Bustillos J, Zhang C, Boesl B, et al. Three-dimensional graphene foam-polymer composite with superior deicing efficiency and strength. ACS Appl Mater Interfaces, 2018, 10: 5022-5029

37 Samad YA, Li Y, Schiffer A, et al. Graphene foam developed with a novel two-step technique for low and high strains and pressuresensing applications. Small, 2015, 11: 2380-2385

38 Boland CS, Khan U, Backes C, et al. Sensitive, high-strain, highrate bodily motion sensors based on graphene-rubber composites. ACS Nano, 2014, 8: 8819-8830

39 Wilde AAM. Proposed diagnostic criteria for the Brugada syndrome: consensus report. Circulation, 2002, 106: 2514-2519

$40 \mathrm{Xu} \mathrm{R}, \mathrm{Lu} \mathrm{Y}$, Jiang C, et al. Facile fabrication of three-dimensional graphene foam/poly(dimethylsiloxane) composites and their potential application as strain sensor. ACS Appl Mater Interfaces, 2014, 6: 13455-13460

41 Zhang M, Wang C, Liang X, et al. Weft-knitted fabric for a highly stretchable and low-voltage wearable heater. Adv Electron Mater, 2017, 3: 1700193

Acknowledgements This work was supported by the National Natural Science Foundation of China (51572025), the National Foundation of China (41422050303), the Program of Introducing Talents of Discipline to Universities (B14003), Beijing Municipal Science Technology Commission and the Fundamental Research Funds for Central Universities.

Author contributions The manuscript was written by $\mathrm{Xu} \mathrm{M}$ with support from all authors. All authors have given approval to the final version of the manuscript.

Conflict of interest The authors declare no conflict of interest.

Supplementary information Experimental details and supplementary data are available in the online version of the paper. 

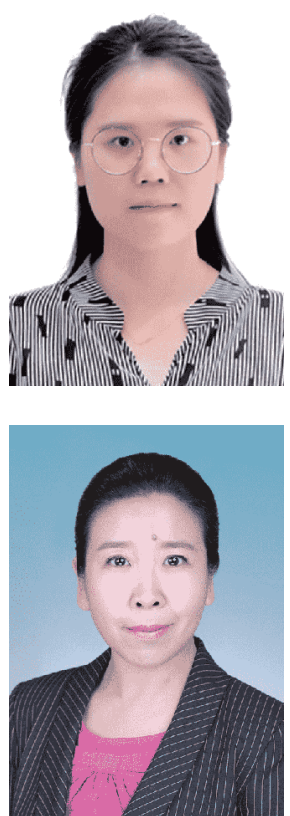

Minxuan Xu received her PhD degree from the Department of Materials Science, University of Science \& Technology Beijing in 2018. She joined the School of Materials and Environmental Engineering at Hangzhou Dianzi University, China as a lecturer in 2018. Her current research focuses on materials and devices for flexible and smart electronics.

Junjie Qi is a full Professor of the University of Science \& Technology Beijing, China. She received her PhD degree from the Department of Materials Science at University of Science \& Technology Beijing in 2002. She has published more than 100 peer review papers. Her current research interest includes the semiconductor nanomaterials, electronic/opto-electronics and the devices of low-dimensional materials.

\section{基于3D泡沫石墨烯的多功能延展性应变传感器用于触觉成像}

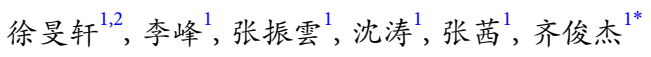

摘要 柔性电子器件的高度发展使得人们对可拉伸应变传感器的综合性能及其多功能化提出了更高的要求. 本文采用一种简单、经济的 方法, 制备了一种基于橡胶、三维石墨烯泡沫和改性硅橡胶的应变传感器. 该传感器具有高延展性和压力、应变传感, 应变可视化以及焦 耳加热等多种特性. 石墨烯泡沫优异的电学性能结合改性硅橡胶理想的机械性能使传感器具有较宽的传感范围(应变检测上限为 $100 \%$, 应 力检测上限为 $66 \mathrm{kPa}$ )、较高的灵敏度 (最大应变灵敏度系数为 584.2 , 最大应力灵敏度系数为 $0.183 \mathrm{kPa}^{-1}$ ) 和较理想的使用寿命 (循环拉伸次 数超过 10000 个周期). 此外, 该传感器在 $5 \mathrm{~V}$ 的直流电压下 5 分钟内可加热 $35^{\circ} \mathrm{C}$, 并可根据温致变色原理使器件的颜色随应变值发生人眼可 见的往复变化, 从而实现应变的可视化. 该柔性多功能应变传感器可作为实时、高精度检测物理刺激的电子皮肤, 热疗或保温的穿戴式加 热器, 或可视化智能触摸面板. 